\title{
Consensus guidelines on analgesia and sedation in dying intensive
} care unit patients

\author{
Laura A Hawryluck*1,2, William RC Harvey ${ }^{3}$, Louise Lemieux-Charles ${ }^{4}$ and \\ Peter A Singer $2,3,5$
}

Address: ${ }^{1}$ Ian Anderson Continuing Education Program In End of Life Care, Toronto; Canada, ${ }^{2}$ University of Health Network, University of Toronto, Toronto, Canada, ${ }^{3}$ University of Toronto Joint Centre for Bioethics, Toronto, ${ }^{4}$ Department of Health Policy, Management and Evaluation, University of Toronto, Toronto, Canada and ${ }^{5}$ Department of Medicine, University of Toronto

Email: Laura A Hawryluck* - laura.hawryluck@utoronto.ca; William RC Harvey - william.harvey@utoronto.ca; Louise LemieuxCharles - l.lemieux.charles@utoronto.ca; Peter A Singer - peter.singer@utoronto.ca

* Corresponding author

Published: 12 August 2002

BMC Medical Ethics 2002, 3:3

This article is available from: http://www.biomedcentral.com/I472-6939/3/3

(C) 2002 Hawryluck et al; licensee BioMed Central Ltd. This is an Open Access article: verbatim copying and redistribution of this article are permitted in all media for any purpose, provided this notice is preserved along with the article's original URL.
Received: 15 April 2002

Accepted: 12 August 2002

\begin{abstract}
Background: Intensivists must provide enough analgesia and sedation to ensure dying patients receive good palliative care. However, if it is perceived that too much is given, they risk prosecution for committing euthanasia. The goal of this study is to develop consensus guidelines on analgesia and sedation in dying intensive care unit patients that help distinguish palliative care from euthanasia.
\end{abstract}

Methods: Using the Delphi technique, panelists rated levels of agreement with statements describing how analgesics and sedatives should be given to dying ICU patients and how palliative care should be distinguished from euthanasia. Participants were drawn from 3 panels: I) Canadian Academic Adult Intensive Care Fellowship program directors and Intensive Care division chiefs ( $N$ = 9); 2) Deputy chief provincial coroners $(\mathrm{N}=5)$; 3) Validation panel of Intensivists attending the Canadian Critical Care Trials Group meeting $(\mathrm{N}=12)$.

Results: After three Delphi rounds, consensus was achieved on 16 statements encompassing the role of palliative care in the intensive care unit, the management of pain and suffering, current areas of controversy, and ways of improving palliative care in the ICU.

Conclusion: Consensus guidelines were developed to guide the administration of analgesics and sedatives to dying ICU patients and to help distinguish palliative care from euthanasia.

\section{Background}

Intensivists are confronted with a difficult balance in providing analgesia and sedation for dying patients. If they administer too little to control distress, they provide inadequate palliative care and patients suffer [1-7]. If they administer too much for life to continue, they may risk prosecution for committing euthanasia [6,8-15]. With re- cently increasing legal scrutiny, fear of prosecution may continue to grow and perpetuate the undertreatment of pain and suffering at the end of life [1-3], [7-16].

The alleviation of pain and suffering is crucial to the provision of quality end-of-life Care [17]. While the US Society for Critical Care Medicine (SCCM) has published 
practice parameters [18] for the provision of analgesia and sedation in critically ill ICU patients, no guidelines exist for analgesic and sedative use in dying patients. In Cana$\mathrm{da}$, the Chief Coroner of Ontario has defined palliative care [19] to help coroners distinguish palliative care from euthanasia; however, these guidelines are not specific to the ICU and give no specific guidance on how to determine whether the doses given were commensurate with patient distress.

The purpose of this study is to develop consensus guidelines on the use of analgesia and sedation in dying ICU patients. Clear guidelines will help develop a standard of care, diminish the risks of under-medication (inadequate palliative care) and over-medication of pain and suffering (possible euthanasia), and thereby improve the overall quality of end-of-life care in the ICU.

\section{Methods \\ Participants}

Three panels were used in this study: The first Delphi panel was formed by approaching the Adult and Pediatric Critical Care fellowship program directors from all of the Canadian academic centers; in academic centres without a Critical Care fellowship program, the Intensive Care Division Chief was approached. Fellowship program directors and division chiefs were chosen since they represent an easily identifiable group of Intensivists who usually act as opinion leaders in Critical Care medicine in their academic centres. The continuing medical education literature has shown the importance of opinion leaders in changing current practice [20]. By using opinion leaders to develop these guidelines, we hoped to increase the likelihood of their eventual dissemination and use. Intensivists in nonacademic centres were excluded in order to ensure panelists were "experts" in critical care medicine. In Canada, non-academic ICUs frequently combine Intensive Care/ Coronary Care Units (ICU/CCU) and may be staffed by physicians who have not been formally trained in Critical Care Medicine. Out of a possible 27 panel members (13 adult, 14 pediatric), 19 (9 adult, 10 pediatric) agreed to participate. Refusals to participate were predominantly attributed to difficulty in fulfilling the time commitments needed to participate in the study ( $\mathrm{n}=2$ adult, $\mathrm{n}=3$ pediatric); no reasons were provided in the remaining cases ( $\mathrm{n}$ $=2$ adult, $\mathrm{n}=1$ pediatric). As the results from the second Delphi round were analyzed, it became clear that a simple consensus statement was not appropriate for both adult and pediatric patients. This paper reports on the adult consensus statement based on the responses of the 9 adult Intensivists: 2 Anesthetists, 4 Respirologists, 2 Internists, and 1 Surgeon; it included 7 men and 2 women.

The second Delphi panel was formed by approaching all of the provincial Deputy Coroners/Medical Examiners. Of a possible 14 panel members, 5 (all men) agreed to participate. Reasons for refusal included lack of time $(n=5)$, and lack of knowledge required to answer the initial Delphi questionnaire $(n=1)$; no reason was given in the remaining cases $(n=3)$. Coroners/medical examiners were consulted to build understanding between the Intensivists and coroners regarding what constitutes good palliative care in the ICU setting and because upon review of a patient's death, coroners/medical examiners are expected to be able to distinguish palliative care from euthanasia in all clinical settings.

Because our goal was to develop consensus guidelines to guide physicians ordering analgesia and sedation for dying patients - and thereby improve the quality of end-oflife care they prescribe - ICU nurses and other allied health professionals were not used in the development of these guidelines.

The third panel was composed of a convenience sample of the 12 academic Adult Intensivists who attended the Endof-Life (EOL) Day of the national Canadian Critical Trials Group (CCCTG) meeting in April 1999. These Intensivists, composing the validation panel, all have an interest in researching the care received by dying patients in the ICU. All 12 EOL Day attendees volunteered to participate. Their responses were used to validate the consensus statements obtained from the original two panels described above. The validation panel was composed of 6 Internists, and 6 Respirologists; it included 8 men and 4 women.

\section{Consensus process}

This study used the Delphi method to develop a consensus statement on the appropriate use of analgesics and sedatives in dying ICU patients. The Delphi method involves several iterations ("rounds") of a questionnaire until consensus is achieved within the chosen panel of "experts" [21-27].

Panelists were provided with background information on the problems of pain control in the ICU; problems in the provision of quality end-of-life care; the importance of a consensus statement on the use of analgesics and sedatives in dying ICU patients; and definitions of palliative care, euthanasia and assisted suicide based on a MEDLINE review of the literature from 1970-1999, prepared by the authors. While definitions of euthanasia and assisted suicide were provided, our goal was not to debate whether these practices should be legalized and literature to support either side of this current debate was not presented to panelists. Responses to the initial Delphi questionnaire were based on this review of the literature and on the "expert" knowledge of the panel members. 
Table I: Initial Delphi Questionnaire ---- Intensivists

I. How should patients' pain and suffering be controlled at the end-of-life? (which drugs?, when?, how much?) Please justify your answer. ${ }^{\text {a }}$

2. What indications of the patient experiencing suffering and/or pain should be used to judge whether an appropriate amount of narcotic or sedative has been used?

3. Is there a maximal dose of narcotic or sedative that you would recommend not be exceeded? Please justify your answer.

4. During withdrawal of care, would you recommend that narcotics and/or sedatives be given in incremental doses once suffering is evident or before it begins?

5. Is euthanasia (as defined in background information) acceptable or unacceptable?

6. a) Is terminal sedation (sedation to relieve pain until death occurs from the disease itself) euthanasia or acceptable practice? b) In terminal sedation, how should the drugs used to induce unconsciousness be incremented to palliate without causing death?

7. How can the intentions of the physicians administering sedatives/narcotics at the end-of-life be assessed (e.g. to palliate vs. to euthanize or to assist suicide)?

8. If the amount of narcotics and/or sedatives required to relieve pain and suffering at the end-of-life may forseeably cause hastening of death although the physician intends only to relieve pain and suffering, should this be considered assisted suicide or euthanasia?

9. How can palliative care be distinguished from euthanasia/assisted suicide in the ICU setting?

a This question was omitted from the coroners' initial questionnaire.

In the first Delphi round, the Intensivist panel was asked a series of 9 open-ended questions describing how analgesics and sedatives should be given to dying ICU patients and how palliative care should be distinguished from euthanasia (Table 1). The coroner/medical examiner panel was asked 8 questions; their questionnaire was the same as the Intensivists', but the first question was omitted because it required "expert" clinical knowledge that the coroners would not possess. Responses to all Delphi rounds were obtained via mail, fax and e-mail.

Since our goal was to develop Intensivist-based consensus guidelines, the second Delphi round involved the Intensivist panel only. The questions and concerns raised by the coroner panel in the first Delphi round were inserted into the appropriate section of the document that had been generated by the Intensivist panel. Intensivist panelists were then asked to comment on the document generated in the first round, clarify any obscurities, and raise any questions not adequately addressed. They were asked to respond to new questions that were generated from either the qualitative analysis of their previous responses, or from the responses of the coroner panel in the first Delphi round. They were also asked to rate their level of agreement with each section and the overall document on a Likert scale ranging from 1 (complete disagreement) to 7 (complete agreement).

The third Delphi round involved both adult Intensivist and coroner panels. A summary of each section of the document generated in the second round - which represented the proposed consensus statement for that section - was inserted as an italicized statement at the beginning of each section. Panelists were then asked to rank their agreement with each consensus statement on a Likert scale, and were asked to identify modifications that would allow them to agree completely with each statement. They were also asked to rank their overall agreement with the document and, again, identify changes that would allow them to agree completely with the document. Median scores from the second round were then presented to panelists in the third Delphi round, enabling individual panel members to compare their level of agreement with that of other panelists. A fourth Delphi round was not required since consensus was achieved in the third round.

The third panel (validation panel) was presented with the final consensus statements obtained after the third Delphi round. They were also asked to rate their agreement with each statement on the same Likert scale. If they gave any statement a score less than 5, they were again asked to identify modifications that would allow them to agree completely with that statement.

\section{Data analysis}

The responses of the Intensivists and coroner panels were analyzed separately. Qualitative analysis was performed by content analysis in each round [27]. Initially open coding was used to give conceptual labels to randomly selected panelist responses [27]. These conceptual labels generated the formation of categories (or sections) $[22,23,27]$. Subsequently, the properties of each category were delineated [27]. The responses of all panel members carried equal weight $[22,27]$.

Since a normal distribution could not be assumed, the median score obtained from each section was used to determine agreement among panelists. It was stipulated that consensus was determined as $80 \%$ agreement (a median Likert score of 5.6 on 7 point scale) within the initial 2 panels (Intensivists and coroners) [21,22]. It was decided a priori that statements which did not achieve a Likert score of 5.6 would be discarded. 
Table 2: Palliative Care in the ICU

Palliative Care in the ICU

Good Intensive Care must seek to provide relief of pain and suffering for ALL Intensive Care Unit (ICU) patients, not solely for those for whom death is inevitable. The palliation of dying patients in the ICU is different from palliative care in other settings since the dying process tends to be more dramatic and the time from withholding/withdrawing active disease treatment to death is much shorter. Ensuring good palliative care in the ICU is crucial.

Goals of Palliative Care in the Intensive Care Unit

The goals of palliative care in the ICU are: I) relief of pain, 2) relief of agitation and anxiety, 3) relief of dyspnea, 4) psychological and spiritual support of patient and family and, 5) provision of comfort by changing the technological ICU environment to a more comfortable, peaceful one. Patients' wishes, including those expressed by advance directives, must be respected by the medical team.

Difficulties in the Assessment of Pain and Suffering

Pain and suffering are different. The ability to assess a patient's pain and suffering is crucial, yet these skills are poorly taught, if taught at all. In the Intensive Care Unit, pain assessment is rendered even more difficult by: I) communication problems imposed by the ICU environment, 2) the severity of illness and the presence of multisystem organ failure, 3) decreased level of consciousness of patients as a result of illness and drugs, 4) our own lack of knowledge/difficulty in interpretation of clinical signs, and, 5) the unreliability of clinical signs. Suffering, because of its even greater individual nature, is harder to assess. Since the assessment of suffering may not be easily amenable to teaching, what must be taught is respect for others' values; values through which individual suffering is perceived. Intensivists need to be aware of the abilities of their ICU staff in assessing and ensuring adequate relief of pain and suffering. Education, research and discussions with family members may be invaluable in improving the abilities of physicians and nurses to determine patient suffering

No modifications were made to the statements as a result of the validation panel's comments. No statements were discarded.

\section{Research ethics}

This study was approved by the University of Toronto's Research Ethics Committee. Informed consent was obtained from each panel member. Responses were kept confidential and, after responses were received by the principal investigator; all identifying information relating to the individual panel member was removed.

\section{Results}

In the third round, consensus was obtained in both Delphi (Intensivist and coroner) panels on 16 statements. These statements can be organized into 4 categories (tables 2,3,4,5). The first category (table 2) discusses 1 ) how palliative care is different in the ICU than in other settings, 2) the role of palliative care in the ICU, and 3) the difficulties in assessing pain and suffering that are specific to the ICU.

The second category of consensus statements (table 3 ) describes the management of pain and suffering in the ICU. These guidelines provide practical guidance on what clinical factors to consider when selecting an initial starting dose of analgesics and sedatives and how to judge whether an effective level of palliation was achieved. In view of the high doses of drugs used in the ICU, the guidelines emphasize that a maximum dose does not exist but rather, the palliation of pain and suffering must be individualized to each dying patient and drugs administered in accordance with clinical need.

The third category of consensus statements (table 4) focuses on areas of controversy in the current literature. These statements discuss the role of palliation in persistently vegetative patients, and describe how dying patients paralyzed with neuromuscular blockers should be managed. According to participants, terminal sedation represents another means of alleviating pain and suffering at the end of life and is not euthanasia. The principle of double effect whereby analgesics and sedatives may be administered to alleviate distress even though death may be hastened by their administration was seen to be useful in allowing physicians to achieve an effective level of analgesia. The physician's intent when administering these drugs was seen as the distinguishing factor between palliative care and euthanasia. Documentation of the reasons for withholding/withdrawing life-sustaining treatments and the ways pain and distress will be alleviated is important. This not only ensures that the physician's intent in ordering and administering analgesics and sedatives is clear, but improves the quality of end-of-life care within the ICU by detailing a clear and comprehensive treatment plan.

Finally, the fourth category of consensus statements (Table 5) discusses how palliative care in the ICU can be improved in the future through research and ongoing education of the staff. The guidelines explore the role of 
Table 3: Management of Pain and Suffering

Relief of Pain and Suffering

In order to relieve pain and suffering at the end-of-life, both pharmacological and non-pharmacological means should be used. Non-pharmacological interventions include ensuring the presence of family, friends and pastoral care (if desired), and, changing the technological ICU environment to a more private and peaceful one. Nursing interventions and accommodating patients' religious and cultural beliefs also play an important role in alleviating pain and suffering. Pharmacological interventions include any analgesics, sedatives or other adjuncts that will decrease discomfort. In general, narcotics are used for pain; benzodiazepines are used for agitation and anxiety. If the patient is experiencing pain and suffering, both analgesics and sedatives are used. This combination of drugs may provide better relief of pain and suffering at the end-of-life than either class of drug alone.

\section{Initial Dosage}

Most ICU patients require narcotics and sedatives in order to ease the pain and suffering associated with their critical illness. The amount of drugs needed varies on an individual basis. As in active disease treatment, palliative care MUST be individualized. Considerations affecting the initial dose of narcotics and sedatives in palliation include: I) the patient's previous narcotic exposure since tolerance develops quickly, 2) age, 3) previous alcohol or drug use and/or abuse, 4) underlying illness, 5) underlying organ dysfunction 6) the patient's current level of consciousness/ sedation, 7) level of available psychological/spiritual support, and, 8) patients' wishes regarding sedation.

Titration of Analgesics and Sedatives

Once analgesics and sedatives are initiated, they are increased in response to I) patient's request, 2) signs of respiratory distress, 3) physiological signs: unexplained tachycardia, hypertension, diaphoresis, 4) facial grimacing, tearing, vocalizations with movements, turns or other nursing care, and 5) restlessness. These clinical indicators, although crucial for graduated therapy, are imprecise. Ramsay or Likert scales, despite their limitations, may provide additional help in evaluating the patient's discomfort. The total amount of drugs required for any individual patient may far exceed any preconceived notions of usual, in reality non-existent, doses.

Does a Maximal Dose Exist?

No maximum dose of narcotics or sedatives exist. The goal of palliative care is to provide relief of pain and suffering and whatever the amount of drugs that accomplishes this goal is the amount that is needed for that individual patient. By refusing to define a maximal dose of analgesics or sedatives, our goal is to ensure that Intensivists will use the required dose for each patient. If a maximal dose is ever declared, some patients will be in pain and will be suffering at the end-of-life because of the Intensivist's fears of litigation if this maximal dose is exceeded. Therefore, the intent of the physician administering the drugs becomes important in distinguishing between palliative care and assisted death (euthanasia/assisted suicide).

Should Analgesics and Sedatives be Administered in Response to Signs and Symptoms of Pain and Suffering, or Before They Begin?

Support for both approaches exists among Intensivists on this panel. The treatment of signs and symptoms of pain and suffering is good palliative care. When appropriate doses of narcotics and sedatives are used and the intent of the physician is clear and well documented, pre-emptive dosing in anticipation of pain and suffering is not euthanasia nor assisted suicide but good palliative care.

emotional and psychological support of the ICU staff to help them deal with the frequency of deaths in critically ill patients. Such support will hopefully increase their abilities to cope with the emotional and psychological stress in caring for critically ill patients and allow them to provide consistently good palliative care. The guidelines propose increasing ties with Palliative Care Medicine. Traditionally considered to be the polar opposite field in the spectrum of care, Palliative Care Medicine may be invaluable in helping to improve the quality of end-of-life care in the ICU setting.

All guidelines achieved high degrees of consensus. The final median Likert scores of all three panels are provided in table 6.

\section{Discussion}

The goal of this study is to develop consensus guidelines on analgesia and sedation that consider the unique challenges encountered when palliating dying ICU patients, and help distinguish palliative care from euthanasia in the ICU setting. The guidelines are not intended to argue for or against the legalization of euthanasia. Our hope is that the guidelines will decrease the confusion and anxiety regarding the use of opiates and sedatives at the end of life and thereby improve the quality of care received by dying patients. To meet this goal, we have divided the guidelines into sections describing palliative care in the ICU setting, management of pain and suffering, areas of controversy, and ways of improving palliative care in the ICU.

A recent review of the literature by Ostermann et al. [28] revealed the paucity of good randomized controlled trials 
Table 4: Current areas of Controversy

\begin{abstract}
Special Situations
Neuromuscular blockers mask the clinical signs of pain and suffering delineated above. When possible, the withholding and withdrawal of life support should be started after their effects wear off in order to permit Intensivists to assess as accurately as possible the patient's pain and suffering and ensure good palliative care. If neuromuscular blockers were not in use, they should not be started in order to hide patient distress. The intent and justifications of Intensivists who fail to wait for neuromuscular blockers to wear off or who fail to reverse them must be carefully documented. Since patients in persistent vegetative states are deemed incapable of feeling pain or anxiety, sedatives and narcotics are usually not required during the withholding/withdrawal of life support. The family's perceptions of pain and suffering, however, may play a role in the use of narcotics and sedatives in these patients.
\end{abstract}

Terminal Sedation

Terminal sedation, defined in the literature as sedation with continuous IV narcotics and/or sedatives until the patient becomes unconscious and death ensues from the underlying illness, is palliative care, not euthanasia. Since terminal sedation may arguably make the detection of euthanasia/ assisted suicide more difficult, the intent of the Intensivist is crucial.

Intent

The intention of the Intensivist administering narcotics/sedatives to palliate dying patients can be assessed by careful documentation in the chart of: I) the patient's medical condition and reasons leading to the initiation of palliative care, 2) the goal, which is to relieve pain and suffering, 3) the way pain and suffering will be evaluated, and 4) the way in which drugs will be increased and why. Intensive care units should develop guidelines governing the process of withholding and withdrawal of life support and Intensivists should justify and document any need to deviate from the policy and the anticipated modifications. The administration of drugs without any palliative benefit, e.g. lethal doses of potassium chloride or neuromuscular blockers, suggests an intent to euthanize/assist in the suicide of an individual patient.

Principle of Double Effect

If the amount of narcotics/sedatives required to relieve pain and suffering at the end-of-life may foreseeably cause hastening of death, although the physician's intent is solely to relieve suffering, this should be considered palliative care.

\title{
Distinction between Palliative Care and Euthanasia
}

The intent of the physician administering narcotics and sedatives to the dying patient is the most crucial distinction between palliative care and assisted death (euthanasia/assisted suicide). In order to avoid any misinterpretations, Intensivists must clearly document, in the patient's chart, their intentions and justify their actions during the withholding/withdrawal process.

on sedation in ICU patients. Research has revealed problems in alleviating pain even among skilled palliative care providers $[1-4,29,30]$ and there is no proof in the current literature that Intensivists are better at relieving their dying patients' distress than other physicians [3]. Healthcare providers withdrawing treatments from dying patients frequently encounter apprehension and concern from surviving family members about how their loved one's pain and distress will be treated. In these situations of withdrawal of life-sustaining treatment, both healthcare providers and family members hope for a speedy, comfortable death in which pain and other distressing symptoms are well controlled and the process of dying is accomplished in peace with loved ones at the bedside [17]. However, questions by family members often reveal concern that their loved one's final moments will be filled with suffering, or, alternatively, that drugs such as opiates and benzodiazepines given by the healthcare team will unduly hasten the dying process and/or kill their loved one [1-3], [7-16,32,33].

Cases of euthanasia committed by healthcare providers have occurred and receive widespread media attention [915]. These highly publicized cases of euthanasia, restrictive prescribing legislation, and increased scrutiny by coroners, licensing bodies, families and the public, lead healthcare providers to under-treat the pain and symptoms of their dying patients [1-16,32,33]. Furthermore, fears of being misunderstood - of having their intent to palliate mistaken for an intent to kill - are currently being exacerbated by debates/articles in the medical literature [34-37]. The authors of these articles seek to promote the legalization of euthanasia by misappropriating ethical principles such as the Principle of Double Effect in order to confuse their colleagues and diminish the fundamental and very real differences between the practices of palliation and euthanasia [34-37]. An unintended conse- 
Table 5: Ways of Improving Palliative Care in the ICU

How Can We Improve our Abilities and our Consistency in Assessing and Treating Pain and Suffering?

Open discussions involving all members of the health care team and family, consulting and sharing when faced with difficult cases, improvements in education and research are needed. The development of a process to review our performance in palliative care within each ICU and national consensus guidelines will also improve our skill in assessing pain and suffering and improve our abilities to relieve it at the end-of-life.

Support for the Intensive Care Unit Staff

The importance of psychological and emotional support for the ICU staff involved in palliating a dying patient is frequently overlooked. Developing a supportive working group, open communication and regular debriefings among members of the ICU team is crucial. The ICU social worker, pastoral care representative and, within the hospital, the departments of psychiatry and psychology may also be very helpful in enabling the ICU staff to continue to provide good palliative care.

Palliative Care Medicine Consultation

Currently a formal Palliative Care consult is rarely requested during the withholding and withdrawal of life support. If the expertise exists within the ICU, such a consult is not required. A Palliative Care Medicine consultation could be useful to: I) treat symptoms that are difficult to control, 2) treat difficult pain syndromes, 3) provide guidance on the use of adjuncts that we, as Intensivists, use infrequently in the dying process, 4) provide guidance when using analgesics/sedatives infrequently administered, 5) help when significant psychological issues within the family or health care team are evident, 6) provide guidance in ICUs where the practices of withholding/withdrawal of care is infrequent, 7) help ease the patient's transfer to the ward if he/she survives the withholding/withdrawal process, and 8) provide ongoing help in relieving pain and suffering when death is protracted.

Table 6: Median Likert Agreement Scores ---- Final Delphi Round

\begin{tabular}{lccc}
\hline & Intensivist & Coroner & Validation \\
\hline Palliative Care in ICU: & $7(5-7)^{\mathrm{a}}$ & $7(5-7)$ & $6(3-7)$ \\
Goals of Palliative Care: & $7(6-7)$ & $6(5-7)$ & $7(4-7)$ \\
Difficulties in Assessment: & $7(6-7)$ & $7(4-7)$ & $6(5-7)$ \\
Relief of Pain \& Suffering: & $7(4-7)$ & $7(6-7)$ & $7(6-7)$ \\
Initial Dose: & $7(4-7)$ & $7(6-7)$ & $6.25(5-7)$ \\
Titration: & $7(6-7)$ & $7(5-7)$ & $5.25(4-7)$ \\
Maximal Dose: & $7(2-7)$ & $7(5-7)$ & $6.5(4-7)$ \\
Timing of Administration: & $7(6-7)$ & $7(5-7)$ & $6(4-7)$ \\
Special Situations: & $7(3-7)$ & $7(5-7)$ & $6(2-7)$ \\
Terminal Sedation: & $7(4-7)$ & $7(4-7)$ & $6(5-7)$ \\
Intent: & $7(4-7)$ & $7(6-7)$ & $6(3-7)$ \\
Double Effect: & $7(6-7)$ & 7 & $7(5-7)$ \\
Palliative Care vs. Euthanasia: & $7(4-7)$ & 7 & $6(4-7)$ \\
Improvement of Palliation: & $7(5-7)$ & $6(5-7)$ & $6(5-7)$ \\
Support for Staff: & $7(5-7)$ & $7(5-7)$ & $6(5-7)$ \\
Palliative Care Consult: & $7(2-7)$ & $7(5-7)$ & $5.5(3-7)$
\end{tabular}

a values in () indicate range of Likert scores

quence may be to increase the reluctance on the part of health care providers to administer adequate analgesia and sedation to dying patients.

The US Society for Critical Care Medicine practice parameters [18] and in Canada, the Ontario Chief Coroner's memo on palliative care [19], have established practice parameters on analgesia and sedation. Unlike our guidelines, the SCCM practice parameters [18] do not discuss the use of opiates and sedatives in dying patients, while the Ontario Chief Coroner's guidelines[19] do not relate to the ICU. Brody et al. [38] also developed guidelines for compassionate management in withdrawing intensive life-sustaining treatments; however, these guidelines were based on a literature review, did not use consensus methodology or involve a national panel of Intensivists and only represented the authors' opinions. Furthermore, Bro- 
dy et al. [38] do not attempt to address how palliative care can be distinguished from euthanasia in the ICU.

To our knowledge, our consensus guidelines on analgesia and sedation in dying ICU patients are the first of their kind developed using consensus methods and involving coroners. The use of consensus methodology and the participation of coroners will hopefully serve to clarify the areas of controversy, their nature and help us resolve these dilemmas in the future. As well, participation by coroners will hopefully result in increased understanding of the difficulties faced both by those caring for the dying and for those asked to review the care received - for it is only through open dialogue that we can ever hope overcome any doubts about intent - to palliate or to kill - and thereby improve the quality of end-of-life care.

This study has three main limitations. First, our guidelines use a small group of expert Intensivists and Coroners to focus attention on how pain and suffering ought to be palliated at the end of life. While our panel sizes were small, saturation of responses occurred in each Delphi round, a sign of credibility (internal validity). Although we attempted to validate our consensus statements by using an independent panel of Intensivists, this panel was also small. While the validation panel was a convenience sample, panelists were representative of Intensivists across the country and agreed with all of the developed consensus statements, a sign of transferability (external validity).

Second, our consensus guidelines are physician-based. They represent an initial attempt to improve the quality of end-of-life care in the ICU by describing best practice in regards to the administration of analgesics and sedatives to dying patients. In order to improve the overall quality of end-of-life care in the ICU, these consensus guidelines need to be incorporated into an overall program to educate the ICU staff in caring for dying patients. These guidelines can then be discussed and modified as needed by other members of the ICU team

Third, our consensus guidelines are developed from a professional and not a patient's perspective. Intensivists' assumptions on how to best treat pain and suffering at the end of life may differ markedly from patients' and families' lived experiences. More research is needed to explore the patient's and family's perspective on how palliative care can be improved in the ICU.

\section{Conclusion}

We have developed a set of guidelines that can be used by critical care societies, training programs, and coroners alike to guide the provision of analgesia and sedation in the ICU. Application of our guidelines should minimize the twin risks of under-treatment (and its resultant pain and suffering) and over-treatment (and its resultant risk of prosecution for euthanasia) for dying patients in the ICU.

\section{Competing interests}

None declared.

\section{Authors' contributorship}

LAH: involved in research planning, design, data collection and analysis, writing of paper, revising of paper, and approving final draft. WRCH: research project design, interpretation of results, critical appraisal of paper drafts, and approval of final draft. LLC: research methods, design, data analysis, critical appraisal of paper drafts, and approval of final draft. PAS: research project concept, design, methods, interpretation of results, actively involved in writing/revising drafts of paper, and approval of final draft.

\section{Acknowledgments}

Dr. Hawryluck was supported by a University of Toronto Postgraduate Medicine Fellowship Award. Dr. Singer is supported by an Investigator award from the Canadian Institutes of Health Research and he is Sun Life Financial Chair in Bioethics at the University of Toronto.

\section{References}

I. Meier DE, Morrison S and Cassel CK Improving palliative care Ann Intern Med 1997, 127(3):225-230

2. Max MB Improving outcomes of analgesic treatment: is education enough. Ann Intern Med 1990, I I 3(I I):885-889

3. Latimer E The Problem of Cancer Pain Drug Protocol 1989, 4(4): $11-18$

4. Von Roenn JH, Cleeland CS, Gonin R, Hatfield AK and Pandya KJ Physicians attitudes and practice in cancer pain management Ann Intern Med 1993, 1 1 9:121-126

5. Fein R Accountability and Quality in End-of Life Care. Institute of Medicine, Approaching Death (Edited by: Field M. \& Cassel C) National Academy Press 1997, 122-153

6. Scott J Palliative Care Does not and Should not Hasten Death, Pain Management 1991, 7:2-7

7. Levetown M Palliative Care in the Intensive Care Unit New Horizons 1998, 6(4):383-397

8. Orentlicher D The Supreme Court and Terminal Sedation. Physician Assisted Suicide: Expanding the Debate. Routledge ed. New York 1998, 301-312

9. Doctor Admitted Baby Girl Killed with Drug Overdose". Calgary Herald AI-2May 25, I99I

10. Wilson D Medical Regulatory Body Opposes Euthanasia. Globe and Mail A8Nov. 28, 1991

II. Mungan C Sentence Suspended in Euthanasia Case. Globe and Mail AI-I IAug. 251992

12. Mercy Killing Draws Review. Calgary Herald A3Jun 201992

13. Doctor Reprimanded for Lethal Injection. Globe and Mail Al5Aug. 17, 1992

14. MD Charged with Murder. Chronicle Herald AI-2May 81997

15. Mercy Probe Doctor Called Caring. Vancouver Sun AI-2Feb. 22 |99|

16. Special Senate Committee on Euthanasia and Assisted Suicide. Of Life and Death 25-36June 1995

17. Singer PA, Martin DK and Kelner M Quality end-of-life care: patient's perspectives. JAMA 1999, 28I:163-168

18. Shapiro BA, Warren J and Egol AB Practice parameters for intravenous analgesia and sedation for adult patients in the ICU: an executive summary. Crit Care Med 1995, 23(9): 1596-1600

19. Young JG Memorandum to ontario coroners - memo a 603. Nov. 29 |99|

20. Oxman AD, Thomson MA, Davis DA and Haynes RB No magic bullets: a systematic review of 102 trials of interventions to improve professional practice. CMAJ 1995, I53:|1423-I43I 
21. Jones $J$ and Hunter $D$ Consensus methods for medical and health services research. $B M J$ I 995,3 I I:376-380

22. Fink A, Kosecoff J, Chassin M and Brook R Consensus Methods: Characteristics and Guidelines for Use. AJPH I 984, 74(9):979983

23. Moscovice I, Armstrong P, Shortell S and Bennett R Health Services Research for Decision Makers: the Use of the Delphi Technique to Determine Health Priorities. J of Health Politics Policy and Law 1988, 2:388-410

24. Woodward C and Chamber LW Guide to Questionnaire Construction and Question Writing. Ottawa, Canadian Public Health Association 1983,

25. Fatout M and Rose $S$ Task Groups in Social Services. Sage Publications 1995, I38-142

26. Sackman H Delphi Critique. MA: DC Health 1975 ,

27. Creswell JW Qualitative Inquiry and Research Design: Choosing among Five Traditions. Sage Publications California 1998, 139 165

28. Ostermann ME, Keenan SP, Seifening RA and Sibbald WJ Sedation in the Intensive Care Unit: a Systematic Review. JAMA 2000, 283(I I): I 45I- I 459

29. Latimer E, McDonald J and Krauser J Towards the Provision of Effective Palliative Care in Ontario. Ont Med Rev 22-28July/Aug. 1998

30. Government of Canada: Special Senate Committee on Euthanasia and Assisted Suicide Of Life and Death I8-35June 1995

31. Wilson WC, Smedira NG, Fink C, McDowell JA and Luce JM Ordering and Administration of Sedatives and Analgesics during the Withdrawal and Withholding of Life Support from Critically III Patients. JAMA 1992, 267(7):949-952

32. Hamill-Ruth RJ and Marohn ML Evaluation of Pain in the Critically III Patient. Crit Care Clinics 1999, I 5(I):35-54

33. Levetown M Palliative care in the Intensive Care Unit. New Horizons 1998, 6(4):383-397

34. Foley K Chapter I0: Pain and Symptom Control in the Dying ICU Patient Managing Death in the ICU (Edited by: Curtis RJ \& Rubenfeld GD) Oxford University Press, New York 200I, 103-I25

35. Analgesia Virtue and the Principle of Double Effect. J Pall Med (Edited by: Hawryluck L, Harvey W) 2000, I6S:S24-30

36. Quill TE, Dresser R and Brock DW The Rule of Double Effect: A Critique of its Role in End-of-Life Decision Making. NEJM | 997, 337(24): 1768-|77|

37. Quill TE, Coombs Lee B, Nunn S and for the University of Pennsylvania Center for Bioethics Assisted Suicide Consensus Panel, Palliative Care Treatments of Last Resort Choosing the Least Harmful Alternative. Ann Int Med 2000, 132:488-493

38. Brody H, Campbell ML, Faber-Langendoen K and Ogle KS Withdrawing Intensive Life Sustaining Treatment-Recommendations for Compassionate Clinical Management. NEJM 1997, 336(9):652-657

\section{Pre-publication history}

The pre-publication history for this paper can be accessed here:

http://www.biomedcentral.com/1472-6939/3/3/prepub
Publish with Biomed Central and every scientist can read your work free of charge

"BioMed Central will be the most significant development for disseminating the results of biomedical research in our lifetime. "

Sir Paul Nurse, Cancer Research UK

Your research papers will be:

- available free of charge to the entire biomedical community

- peer reviewed and published immediately upon acceptance

- cited in PubMed and archived on PubMed Central

- yours - you keep the copyright
BioMedcentral 